\title{
In situ cryo-electron tomography: a post-reductionist approach to structural biology
}

Shoh Asano ${ }^{a}$, Benjamin D. Engel ${ }^{a}$, Wolfgang Baumeister ${ }^{a, 1}$

${ }^{a}$ Department of Molecular Structural Biology, Max Planck Institute of Biochemistry; Am Klopferspitz 18, 82152 Martinsried, Germany.

${ }^{1}$ Corresponding author: baumeist@biochem.mpg.de.

Telephone: +49 (89) $8578-2642$

Fax: +49 (89) $8578-2641$

\section{Highlights:}

- Cryo-electron tomography visualizes native frozen cells with molecular resolution

- Protein complexes are identified and characterized within the cellular environment

- Sample preparation and computational approaches are described in depth

- Two recent studies provide examples for characterizing known and unknown complexes

- The in situ structural analysis of every macromolecule in the cell is within reach

Keywords: cryo-EM, tomography, template matching, visual proteomics, subtomogram averaging 


\section{Abstract}

Cryo-electron tomography (cryo-ET) is a powerful technique that can faithfully image the native cellular environment at nanometer resolution. Unlike many other imaging approaches, cryoET provides a label-free method of detecting biological structures, relying on the intrinsic contrast of frozen cellular material for direct identification of macromolecules. Recent advances in sample preparation, detector technology, and phase plate imaging have enabled the structural characterization of protein complexes within intact cells. Here, we review these technical developments and outline a detailed computational workflow for in situ structural analysis. Two recent studies are described to illustrate how this workflow can be adapted to examine both known and unknown cellular complexes. The stage is now set to realize the promise of visual proteomics - a complete structural description of the cell's native molecular landscape. 


\section{Introduction}

Understanding complex networks requires detailed knowledge about the properties and interactions of each of the network's components. This is especially true in molecular cell biology, where individual proteins are often involved in multiple cellular processes, frequently swapping interaction partners and changing states of assembly ${ }^{1,2}$. Structural biologists have traditionally approached this cellular complexity in a reductionist manner by characterizing purified molecular components ${ }^{3}$. This "divide and conquer" strategy has been highly successful, as evidenced by the impressive number of entries in the Protein Data Bank ${ }^{4}$. However, the reductionist approach has several intrinsic limitations. Labile and membrane-embedded complexes can be easily damaged or distorted from their functional conformations during purification. Moreover, complexes frequently lose their biologically-relevant associations once they are removed from the crowded cellular environment. This is well known in protein crystallography, as packing forces and missing cofactors can stabilize non-physiological structures ${ }^{5}$.

Cryo-ET avoids these pitfalls by imaging unperturbed cells, preserving the full spectrum of each molecule's conformations and interactions ${ }^{6-8}$. With several recent technological advances increasing both the versatility and the image quality of cryo-ET, it is now possible to identify and characterize macromolecules within a wide variety of cell types ${ }^{9-16}$. Computational tools including template matching, subtomogram averaging, and classification and enable the generation of molecular-resolution structures that accurately represent the physiological conformations of complexes within their functional environments ${ }^{17-19}$. In this review, we present a practical workflow for in situ structural analysis, with an eye towards the future of visual proteomics ${ }^{20,21 .}$ 


\section{Preparation of thin vitreous samples and cryo-ET data acquisition}

Sample preparation for cellular cryo-ET follows a relatively straightforward workflow that is compatible with most cell types (fig. 1). Cells that grow in solution, including bacteria, archaea, yeast, and many protists, are cultured in their standard medium and applied to carbon-coated copper EM grids just prior to freezing (figs. 1a and b) ${ }^{10,22-25}$. Alternatively, adherent cells, such as Dictyostelium, mammalian cell lines, and neurons, are grown on top of carbon-coated gold EM grids, which are less cytotoxic than copper (fig. 1a) ${ }^{11,12,26-28}$. The cells are then vitrified in glass-like amorphous ice, which is free of damaging ice crystals and does not discretely scatter the electron beam, facilitating cryo-ET imaging ${ }^{29,30}$. Vitrification immobilizes the sample, while preserving native molecular structures, maintaining a hydrated state, and decreasing the radiation sensitivity to incident electrons ${ }^{29,31}$.

Vitrification of cells is commonly performed by one of two methods, plunge freezing or highpressure freezing (HPF) (fig. 1b). Plunge freezing is suitable for cells up to $10 \mu \mathrm{m}$ in diameter $29,32,33$. Equipment for manual plunge freezing is now readily accessible, with homemade designs providing a cost-effective setup ${ }^{34}$. Automated plunge freezing devices enable control of humidity, temperature, blotting strength, and blotting time, improving sample reproducibility ${ }^{35,36}$. Alternatively, HPF is capable of freezing multicellular organisms and tissue up to $200 \mu \mathrm{m}$ in thickness ${ }^{37,38}$. However, subsequent thinning is mandatory and usually performed with a cryo-ultramicrotome, which cuts vitreous sections that are susceptible to compression and crevasse deformations ${ }^{39-41}$. A few strategies have been developed to avoid these artifacts when thinning HPF material, including a liftout approach combining cryo-focused ion beam (FIB) milling with a cryo-micromanipulator ${ }^{42-44}$.

Once vitreous cellular material is on the EM grid, an optional next step is to localize structures of interest using correlative fluorescence microscopy (FM) (fig. 1c, top). Fluorescentlylabeled molecules within the frozen cells are imaged by cryo-FM, and this information is subsequently used to locate positions of interest on the EM grid inside the electron microscope ${ }^{45-49}$. 
Adapting the recent advances in "super-resolution" FM to cryogenic samples would greatly increase the precision of correlation ${ }^{50,51}$.

While some prokaryotic cells and eukaryotic cellular protrusions are small enough to be imaged whole by cryo-ET ${ }^{9,11,23,24,27,52}$, most cells must be thinned to render them transparent to the electron beam. Cryo-FIB milling (fig. 1c, bottom) is the method of choice, enabling compressionfree thinning of samples directly on the same grid that will be used for tomography ${ }^{53-55}$. Gallium ions are used to ablate material to any specified thickness, typically producing $100-300 \mathrm{~nm}$-thick windows into the native cellular interior ${ }^{56,57}$.

After thinning, the grids are transferred into the transmission electron microscope and tiltseries images are acquired (fig. 1d). These images are then aligned and reconstructed to generate 3D views of the sample, called tomograms. Frozen biological material is easily damaged by electron radiation ${ }^{58-61}$. A common rule of thumb is that tilt-series acquisition should not exceed $100 \mathrm{e}^{-} / \AA^{2}$, although the dose limit varies with specimen type and may be substantially lower to preserve highresolution information ${ }^{59,60,62-64}$. This radiation sensitivity places constraints on many cryo-ET acquisition parameters, including the magnification (pixel size), the tilt scheme, and the amount of signal to noise in the images ${ }^{58}$. As a result, cryo-tomograms are often noisy and have low contrast, presenting challenges to tilt-series alignment and identifying macromolecules within the tomogram volumes. Thicker cellular volumes require higher electron dose to yield the same amount of image signal, further limiting cryo-ET acquisition.

Two recent technical developments, direct electron detectors and the Volta phase plate, have dramatically improved the image quality of cryo-ET ${ }^{65-68}$. Direct electron detectors are complementary metal-oxide-semiconductor (CMOS)-based cameras that convert electrons directly into digital signals ${ }^{69}$. Their major advantages over charged couple devices (CCDs) include higher sensitivity that enables smaller pixel sizes at the same electron dose, improved detection precision that produces sharper images, and a fast frame readout that allows for image drift correction ${ }^{70,71}$. 
Several studies have demonstrated the potential of these new cameras for single-particle cryo-EM, yielding sub-nanometer-resolution protein structures ${ }^{65,67,72-77}$ and heralding the beginning of the cryo-EM "resolution revolution" 78,79 . The gains of applying direct electron detectors to cryo-ET have been similarly impressive $9,15,80-83$, although there is still substantial room for resolution improvement in computational steps such as tilt-series alignment.

Previously, the only way to improve the contrast of cryo-EM images without increasing the electron dose was to use a higher defocus. However, this limits the image resolution, as there is a negative correlation between defocus and resolution. The Volta phase plate decouples this relationship by providing visually impressive contrast during in-focus acquisition, thus producing images of both high contrast and high resolution ${ }^{9,10,15,68}$. This boost in contrast provides major advantages for both tilt-series alignment and structure identification.

\section{Detection of macromolecules inside cells}

Several consecutive image processing steps are necessary for the detection of macromolecules within cellular tomograms (fig. 2). Tilt-series that were recorded with defocus can be subjected to contrast transfer function (CTF) correction to increase their resolution ${ }^{58,84,85}$. This step is unnecessary for in-focus images acquired with the Volta phase plate. Denoising procedures (fig. 2a), such as bandpass frequency filtering, non-local means filtering, and iterative reconstruction schemes ${ }^{86-92}$, can be performed during or after tomogram reconstruction. Proper denoising can aid in tomogram interpretation, and is particularly useful for the segmentation of supramolecular structures and organelles ${ }^{11,93,94}$.

While tomogram segmentation (fig. $2 \mathrm{~b}$ ) is optional, there are several reasons to mask cellular compartments. Segmentation can be utilized to selectively search for macromolecules inside specific organelles or the cytoplasm ${ }^{9,16,95}$. Restricting the search volume to the cell body also 
reduces false positives ${ }^{9,95}$, excluding gold fiducial markers and grid contaminants that are often assigned high scores by template matching (fig. 2c) ${ }^{96-98}$. Tomogram segmentation can be performed either manually or automatically, with several toolboxes available ${ }^{99}$. The latest automatic segmentation algorithms produce accurate results, which can be refined by providing boundary conditions ${ }^{94,100}$. However, manual segmentation may still be required for more complex cellular architecture that requires interpretation and a priori knowledge ${ }^{10,94,100}$.

Template matching (fig. 2c) is the computational method of searching for molecular complexes inside tomographic volumes ${ }^{101,102}$. A template is created using known information about a macromolecule, such as its crystal structure, rough morphology, or known binding partners ${ }^{101}$. As described later in this review, templates may also be generated de novo from the tomogram (Fig. $4)^{16}$. The template matching algorithms perform cross-correlation calculations to compare the template with each position in the tomogram, resulting in a correlation volume where each pixel is assigned a similarity score $20,102,103$. Subvolumes corresponding to the highest scores are then extracted from the tomogram and can be manually screened prior to further analysis to remove obvious false positives $^{9,16,104,105}$.

Once the subvolumes have been extracted, they are subjected to subtomogram averaging (fig. 2d). Averaging methods use cross-correlation measurements to attempt to maximize the spatial overlap between all the subvolumes and the reference volume. Multiple rounds of iterative optimization are performed; each round yields a more refined subtomogram average, which in turn is used as a reference for the next round of alignment ${ }^{19,106,107}$. Different methods have been developed to perform subtomogram averaging in either real space or orthogonal spaces ${ }^{105-107}$.

Unlike purified complexes, macromolecules within the native cellular environment may show a high degree of heterogeneity in their conformations, interaction partners, and states of assembly. Thus, all initial subtomogram averages should be subjected to an additional classification step (fig. 2e) to divide the dataset into more homogeneous subsets ${ }^{9,19,108}$. During classification, 
scoring functions iteratively sort the subvolumes into classes based on relative similarity ${ }^{18,105,109}$. The recently developed autofocused 3D classification algorithm reduces human interaction to a minimum by using the differences within a group of aligned subvolumes, given by the grey-value variance of their average, to automatically generate masks for the scoring function ${ }^{18}$. Even subtle assembly and conformational differences can be reliably detected and separated by this algorithm ${ }^{18,}$ 108. Additional rounds of classification may be applied to create even more homogenous subclasses, provided that there are still enough subvolumes remaining to allow for proper alignment ${ }^{9,108}$. Finally, rigid body or flexible fitting can be used to dock atomic structures of subunits into the classified averages ${ }^{110-112}$, aiding in the characterization of different conformations or assembly states ${ }^{9,113}$.

Two major concerns that need to be taken into consideration during the averaging of both single particles and subtomograms are anisotropic resolution and model bias. An inhomogeneous distribution of orientations in the dataset causes anisotropic resolution, where some regions of the final average are better resolved than others. This phenomenon is most commonly encountered in single-particle cryo-EM ${ }^{114}$, where isolated complexes sometimes assume similar orientations in the thin ice of the EM grid. In situ cryo-ET has the advantage that complexes suspended within the cellular volume usually do not have preferred orientations, and even if they do, these orientations will vary between cells. Therefore, subtomogram averaging can produce relatively isotropic structures from only a moderate number of in situ subvolumes $9,108,115$.

Averaging a large dataset of pure noise images can accurately reproduce the structure of the reference used for alignment, a phenomenon known as model bias ${ }^{116-118}$. Single-particle cryo-EM studies may be especially prone to this artifact, as high-resolution structures are generated from near-focus images with limited contrast, making structural features nearly indistinguishable from noise ${ }^{79}$. Model bias can be minimized in cryo-ET by using the Volta phase plate to boost lowfrequency contrast, low-pass filtering the template before searching the tomogram, template 
matching with a truncated structure (e.g. a single-capped 265 proteasome), and initially aligning the subvolumes to a spherical or random reference (fig. 3a) ${ }^{79,115}$.

In addition to the generation of subtomogram averages, in situ cryo-ET presents the unique opportunity to place these structures into their cellular context (fig. 2f). Template matching identifies molecular positions and orientations with sub-nanometer precision, enabling the assignment of each macromolecule to its cellular location. This exact determination of origin is unmatched by biochemical fractionation; for example, cryo-ET reliably discriminates between ribosomes bound to the nuclear envelope and ribosomes bound to the endoplasmic reticulum. The spatial information from template matching can be combined with tomogram segmentation to determine the precise concentrations of molecular complexes within defined cellular compartments $9,11,108$. Template matching can also be used to analyze the clustering of macromolecules, as well as the positioning of macromolecules relative to other complexes, membranes, or the cytoskeleton. Mapping the in situ distributions of macromolecules in different conformations or assembly states may provide important insights into the spatial regulation of protein function inside the cell. Importantly, the plotting of positions and orientations can be expanded to include multiple different types of macromolecules within the same volume, creating interaction maps of the native cellular environment. Further expanding this analysis to the description of all detectable molecular complexes is the goal of visual proteomics ${ }^{20,119}$, which will be discussed in the final section.

\section{In situ characterization of known complexes: 265 proteasomes in neurons}

A recent study characterizing 265 proteasomes in neurons showcases the strength of in situ subtomogram averaging and classification ${ }^{9}$. In this section, we outline this study's computational workflow, which can be adapted to the investigation of other complexes with known structures (fig. 3). The $26 \mathrm{~S}$ proteasome is a macromolecular complex responsible for the regulated degradation of 
proteins that are misfolded, damaged, or no longer needed ${ }^{120,121}$. These protein substrates are marked for destruction by the covalent attachment of ubiquitin and subsequently bind to the $19 \mathrm{~S}$ regulatory particle (RP), a subcomplex of the $26 \mathrm{~S}$ holoenzyme that caps the $20 \mathrm{~S}$ core particle ${ }^{121,122}$. The substrates then undergo ATP-dependent unfolding and are fed into the $20 \mathrm{~S}$ particle, a barrelshaped chamber containing proteolytic degradation sites ${ }^{121,122}$.

To visualize the $26 \mathrm{~S}$ proteasomes in situ, thin regions of cultured mammalian neurons were imaged by cryo-ET with the Volta phase plate. The single-particle cryo-EM structure of the $26 \mathrm{~S}$ proteasome ${ }^{123}$ was then used to generate a low-pass filtered single-capped $26 \mathrm{~S}$ model for template matching. Both the large size (2.5 MDa) and unique shape of the $26 \mathrm{~S}$ holocomplex aided in its identification. To eliminate the possibility of model bias, the first round of subtomogram averaging used a neutral initial reference that was generated by randomly averaging all of the subvolumes (fig. $3 a$, left ${ }^{106}$. The data-driven alignment converged remarkably fast, where the $6^{\text {th }}$ iteration already displayed a low-resolution, single-capped $26 \mathrm{~S}$ proteasome. By the $20^{\text {th }}$ iteration, no major changes in the alignment could be observed, resulting in an average of the holoenzyme with a single clearly visible RP on top of the $20 \mathrm{~S}$ core particle (fig. 3a, right).

The variance map calculated from the average (fig. 3b, right) indicated a major structural component at the position of the second RP (fig. 3b, green circle). The first round of classification was able to identify the cause of this heterogeneity: the dataset consisted of two different assembly states, the more abundant single-capped and less frequent double-capped $26 \mathrm{~S}$ proteasomes (fig. 3b, green inset). The ratio of these two assembly states was consistent studies with previous observations of isolated $26 \mathrm{~S}$ complexes ${ }^{113,124-126}$.

Using a similar strategy, the dataset was subjected to another classification aimed at disentangling the highly variable 19S RP complex (fig. 3b, red circles and red inset). Variances in the average were traced to two major classes that showed significant conformational differences. To address the functions of these classes, the two in situ conformational states were compared with 
atomic models of the $19 \mathrm{~S} \mathrm{RP}$ previously acquired from single-particle cryo-EM (fig. 3d) $)^{113}$. Using rigid body fitting, both in situ conformations were successfully assigned a functional role. Whereas one class matched the 19S RP not bound to substrate (ground state), the second class had a conformation that resembled the 19S RP during substrate commitment or translocation (substrateprocessing state). This class also displayed a large density not present in the single-particle structure that likely corresponded to substrates or proteasome-interacting proteins. This was the first study to successfully localize the $26 \mathrm{~S}$ proteasome in situ, enabling structural characterization of native structures at the single protein level. These insights the may have implications for neuronal function, as the $26 \mathrm{~S}$ proteasome plays a major role in numerous synaptic processes, and its malfunction is linked to several neurodegenerative diseases ${ }^{127,128}$.

\section{De novo analysis of unknown structures: Golgi intracisternal protein arrays}

In situ subtomogram averaging is also capable of characterizing previously unknown structures. In a recent cryo-ET study, intracisternal protein arrays were observed within the Golgi apparatus of FIB-milled Chlamydomonas reinhardtii cells (fig. 4a) ${ }^{16}$. These structures were both abundant and highly regular, enabling de novo structure generation.

The initial template was a single low-pass filtered subvolume containing two cisterna membranes connected by a protein array (fig. $4 \mathrm{~b}$, top). After the first round of template matching, candidate subvolumes were extracted, manually inspected, and averaged. The resulting subtomogram average was subsequently low-pass filtered and used as a refined template to repeat the template matching (fig. 4b). This iterative template generation improved the detection of the protein arrays, revealing an asymmetric structure linking the two membranes, with alternating long and short proteins projecting into the cisterna lumen (figs. $4 \mathrm{~b}$ and c). The structure also showed periodicity along the cisterna membrane, which was measured by performing autocorrelation of the 
average (fig. 4c). Using this information, the subtomogram average was further refined to account for translational symmetry (fig. 4c).

The array's intracisternal projections are most likely interlinked protein complexes that hold the two cisterna membranes together by zipper-like interactions. These interactions appear to determine the narrow spacing of the central cisterna regions (fig. 4d), and thus may force cargo that is transiting the Golgi towards the bud sites at the cisterna periphery. Plotting the positions and orientations of the array-containing subvolumes in two tomograms revealed that all the arrays were asymmetric and most of them were facing the same direction. This observation has big implications for Golgi biology, as it implies that the two membranes of the same cisterna have different compositions. The most promising putative candidates for the arrays are glycosyltransferases, which have compatible structures and have shown been to form homo- and hetero-oligomeric complexes in the Golgi ${ }^{129}$. If the arrays are indeed composed of glycosyltransferases, the cooperative assembly of these proteins into higher-order structures may affect their enzymatic activity. While this study provided the first insights into intracisternal arrays and their possible biological functions, additional work is required to further characterize these structures and determine their molecular identity.

\section{New frontiers for visual proteomics}

The functions of cellular complexes did not evolve in isolation. Thus, we believe the holy grail of structural biology should be to directly observe molecular structures within their functional environments. Thanks to advances in sample preparation and cryo-ET, this vision is starting to become a reality. Although current studies have only examined a few types of macromolecules, extrapolating the in situ approach to its natural conclusion brings us to the concept of visual proteomics: the parallel identification and characterization of every molecular complex inside the

cell. Visual proteomics is not a new idea ${ }^{20,21,119}$, but until recently, researchers lacked the data 
quality and computational power to put this idea into practice. With continuing advances on both fronts, we are now approaching a convergence of cryo-ET and image analysis where it will be possible to analyze the positions, conformations, and interactions of many macromolecules within a single cell through direct visualization ${ }^{20,21}$.

What is possible when cell biology enters the age of visual proteomics? First and foremost, we may discover a new world of macromolecular and supramolecular structures, which have been missed by techniques that rely on biochemical purification or labeling of known proteins. We already see evidence that this may be the case, as the Golgi intracisternal arrays could only be identified by in situ cryo-ET. Visual proteomics will also mark the dawn of "big data" in structural biology. Cellular cryo-tomograms have exceptionally high information density. New computational tools will be required to effectively mine this information, revealing the relationships of every macromolecule with each other and with the local cellular environment. In addition to advances in image processing, bioinformatic and statistical approaches may prove useful for building interaction networks from dense landscapes of visual information.

When the hardware and software of in situ cryo-ET become high-throughput and readily accessible to the majority of researchers, we anticipate that this technique be integrated into the core methodology of cell biology. Biochemistry provides many indirect ways to probe binary molecular interactions. Yet while this approach can successfully dissect molecular function, it lacks cellular context and can be prone to artifacts, requiring in vivo validation. Fluorescence microscopy, with several recent advances in sub-diffraction limit imaging, is invaluable for tracking the dynamics and spatial relationships of known proteins in living cells. However, this mode of visualization lacks molecular resolution and can only simultaneously examine a few specifically-labeled molecular species. In situ cryo-ET complements both of these traditional approaches by providing a complete molecular-resolution view of all macromolecular species within the unperturbed cellular environment. 


\section{Acknowledgements}

This work was supported by an Alexander von Humboldt Foundation postdoctoral fellowship (to

B.D.E.), the European Commission's grant agreement ERC-2012-SyG_318987-ToPAG, and the

Deutsche Forschungsgemeinschaft Excellence Clusters CIPSM and SFB 1035.

\section{References}

1. Berendsen, H. J. \& Hayward S. (2000). Collective protein dynamics in relation to function. Current opinion in structural biology $10,165-9$.

2. Frauenfelder, H., Sligar S. G. \& Wolynes P. G. (1991). The energy landscapes and motions of proteins. Science 254, 1598-603.

3. Gierasch, L. M. \& Gershenson A. (2009). Post-reductionist protein science, or putting Humpty Dumpty back together again. Nat Chem Biol 5, 774-7.

4. Sali, A., Glaeser R., Earnest T. \& Baumeister W. (2003). From words to literature in structural proteomics. Nature 422, 216-25.

5. Durbin, S. D. \& Feher G. (1996). Protein crystallization. Annu Rev Phys Chem 47, 171-204.

6. Lucic, V., Rigort A. \& Baumeister W. (2013). Cryo-electron tomography: the challenge of doing structural biology in situ. The Journal of cell biology 202, 407-19.

7. Vanhecke, D., Asano S., Kochovski Z., Fernandez-Busnadiego R., Schrod N., Baumeister W., et al. (2011). Cryo-electron tomography: methodology, developments and biological applications. Journal of microscopy 242, 221-7.

8. Medalia, O., Weber I., Frangakis A. S., Nicastro D., Gerisch G. \& Baumeister W. (2002).

Macromolecular architecture in eukaryotic cells visualized by cryoelectron tomography. Science 298, 1209-13.

9. Asano, S., Fukuda Y., Beck F., Aufderheide A., Forster F., Danev R., et al. (2015). Proteasomes. A molecular census of $26 \mathrm{~S}$ proteasomes in intact neurons. Science $347,439-42$.

10. Engel, B. D., Schaffer M., Kuhn Cuellar L., Villa E., Plitzko J. M. \& Baumeister W. (2015). Native architecture of the Chlamydomonas chloroplast revealed by in situ cryo-electron tomography. eLife 4.

11. Jasnin, M., Asano S., Gouin E., Hegerl R., Plitzko J. M., Villa E., et al. (2013). Three-dimensional architecture of actin filaments in Listeria monocytogenes comet tails. Proceedings of the National Academy of Sciences of the United States of America 110, 20521-6.

12. Brandt, F., Carlson L. A., Hartl F. U., Baumeister W. \& Grunewald K. (2010). The threedimensional organization of polyribosomes in intact human cells. Mol Cell 39, 560-9.

13. Leforestier, A., Lemercier N. \& Livolant F. (2012). Contribution of cryoelectron microscopy of vitreous sections to the understanding of biological membrane structure. Proc Natl Acad Sci U S A 109, 8959-64.

14. Al-Amoudi, A., Diez D. C., Betts M. J. \& Frangakis A. S. (2007). The molecular architecture of cadherins in native epidermal desmosomes. Nature 450, 832-7.

15. Fukuda, Y., Laugks U., Lucic V., Baumeister W. \& Danev R. (2015). Electron cryotomography of vitrified cells with a Volta phase plate. Journal of structural biology 190, 143-54. 
16. Engel, B. D., Schaffer M., Albert S., Asano S., Plitzko J. M. \& Baumeister W. (2015). In situ structural analysis of Golgi intracisternal protein arrays. Proceedings of the National Academy of Sciences of the United States of America.

17. Bohm, J., Frangakis A. S., Hegerl R., Nickell S., Typke D. \& Baumeister W. (2000). Toward detecting and identifying macromolecules in a cellular context: template matching applied to electron tomograms. Proceedings of the National Academy of Sciences of the United States of America 97, 14245-50.

18. Chen, Y. X., Pfeffer S., Fernandez J. J., Sorzano C. O. S. \& Forster F. (2014). Autofocused 3D Classification of Cryoelectron Subtomograms. Structure 22, 1528-37.

19. Briggs, J. A. G. (2013). Structural biology in situ - the potential of subtomogram averaging. Current opinion in structural biology 23, 261-7.

20. Forster, F., Han B. G. \& Beck M. (2010). Visual Proteomics. Method Enzymol 483, 215-43.

21. Robinson, C. V., Sali A. \& Baumeister W. (2007). The molecular sociology of the cell. Nature 450, 973-82.

22. Grimm, R., Singh H., Rachel R., Typke D., Zillig W. \& Baumeister W. (1998). Electron tomography of ice-embedded prokaryotic cells. Biophys J 74, 1031-42.

23. Murphy, G. E., Leadbetter J. R. \& Jensen G. J. (2006). In situ structure of the complete Treponema primitia flagellar motor. Nature 442, 1062-4.

24. Briegel, A., Ortega D. R., Tocheva E. I., Wuichet K., Li Z., Chen S., et al. (2009). Universal architecture of bacterial chemoreceptor arrays. Proceedings of the National Academy of Sciences of the United States of America 106, 17181-6.

25. Winey, M., Mamay C. L., O'Toole E. T., Mastronarde D. N., Giddings T. H., Jr., McDonald K. L., et al. (1995). Three-dimensional ultrastructural analysis of the Saccharomyces cerevisiae mitotic spindle. The Journal of cell biology 129, 1601-15.

26. Garvalov, B. K., Zuber B., Bouchet-Marquis C., Kudryashev M., Gruska M., Beck M., et al. (2006). Luminal particles within cellular microtubules. The Journal of cell biology 174, 759-65.

27. Lucic, V., Kossel A. H., Yang T., Bonhoeffer T., Baumeister W. \& Sartori A. (2007). Multiscale imaging of neurons grown in culture: from light microscopy to cryo-electron tomography. Journal of structural biology 160, 146-56.

28. Patla, I., Volberg T., Elad N., Hirschfeld-Warneken V., Grashoff C., Fassler R., et al. (2010).

Dissecting the molecular architecture of integrin adhesion sites by cryo-electron tomography. Nature cell biology 12, 909-15.

29. Dubochet, J., Adrian M., Chang J. J., Homo J. C., Lepault J., McDowall A. W., et al. (1988). Cryoelectron microscopy of vitrified specimens. Quarterly reviews of biophysics $21,129-228$.

30. Dubochet, J., Lepault J., Freeman R., Berriman J. A. \& Homo J. C. (1982). Electron-Microscopy of Frozen Water and Aqueous-Solutions. J Microsc-Oxford 128, 219-37.

31. Taylor, K. A. \& Glaeser R. M. (1976). Electron microscopy of frozen hydrated biological specimens. J Ultrastruct Res 55, 448-56.

32. Sartori, N., Richter K. \& Dubochet J. (1993). Vitrification Depth Can Be Increased More Than 10Fold by High-Pressure Freezing. Journal of microscopy 172, 55-61.

33. Studer, D., Graber W., Al-Amoudi A. \& Eggli P. (2001). A new approach for cryofixation by highpressure freezing. Journal of microscopy 203, 285-94.

34. Cole, R., Matuszek G., See C. \& Rieder C. L. (1990). A simple pneumatic device for plunge-freezing cells grown on electron microscopy grids. J Electron Microsc Tech 16, 167-73.

35. Bellare, J. R., Davis H. T., Scriven L. E. \& Talmon Y. (1988). Controlled environment vitrification system: an improved sample preparation technique. J Electron Microsc Tech 10, 87-111.

36. Iancu, C. V., Tivol W. F., Schooler J. B., Dias D. P., Henderson G. P., Murphy G. E., et al. (2006). Electron cryotomography sample preparation using the Vitrobot. Nature Protocols 1, 2813-9. 37. Kanno, H., Speedy R. J. \& Angell C. A. (1975). Supercooling of Water to $-92^{\circ}$ under Pressure. Science 189, 880-1. 
38. McDonald, K., Schwarz H., Muller-Reichert T., Webb R., Buser C. \& Morphew M. (2010). "Tips and tricks" for high-pressure freezing of model systems. Methods Cell Biol 96, 671-93.

39. Al-Amoudi, A., Studer D. \& Dubochet J. (2005). Cutting artefacts and cutting process in vitreous sections for cryo-electron microscopy. J Struct Biol 150, 109-21.

40. Dubochet, J., Zuber B., Eltsov M., Bouchet-Marquis C., Al-Amoudi A. \& Livolant F. (2007). How to "read" a vitreous section. Method Cell Biol 79, 385-406.

41. Han, H. M., Zuber B. \& Dubochet J. (2008). Compression and crevasses in vitreous sections under different cutting conditions. Journal of microscopy 230, 167-71.

42. Hayles, M. F., de Winter D. A. M., Schneijdenberg C. T. W. M., Meeldijk J. D., Luecken U., Persoon H., et al. (2010). The making of frozen-hydrated, vitreous lamellas from cells for cryo-electron microscopy. Journal of structural biology 172, 180-90.

43. Hsieh, C., Schmelzer T., Kishchenko G., Wagenknecht T. \& Marko M. (2014). Practical workflow for cryo focused-ion-beam milling of tissues and cells for cryo-TEM tomography. J Struct Biol 185, 3241.

44. Mahamid, J., Schampers R., Persson H., Hyman A. A., Baumeister W. \& Plitzko J. M. (2015). A focused ion beam milling and lift-out approach for site-specific preparation of frozen-hydrated lamellas from multicellular organisms. Journal of structural biology in Press.

45. Sartori, A., Gatz R., Beck F., Rigort A., Baumeister W. \& Plitzko J. M. (2007). Correlative microscopy: bridging the gap between fluorescence light microscopy and cryo-electron tomography. Journal of structural biology 160, 135-45.

46. Agronskaia, A. V., Valentijn J. A., van Driel L. F., Schneijdenberg C. T. W. M., Humbel B. M., Henegouwen P. M. P. V. B. E., et al. (2008). Integrated fluorescence and transmission electron microscopy. Journal of structural biology 164, 183-9.

47. Schorb, M. \& Briggs J. A. G. (2014). Correlated cryo-fluorescence and cryo-electron microscopy with high spatial precision and improved sensitivity. Ultramicroscopy 143, 24-32.

48. Kukulski, W., Schorb M., Welsch S., Picco A., Kaksonen M. \& Briggs J. A. G. (2011). Correlated fluorescence and $3 \mathrm{D}$ electron microscopy with high sensitivity and spatial precision. Journal of Cell Biology 192, 111-9.

49. van Driel, L. F., Valentijn J. A., Valentijn K. M., Koning R. I. \& Koster A. J. (2009). Tools for correlative cryo-fluorescence microscopy and cryo-electron tomography applied to whole mitochondria in human endothelial cells. Eur J Cell Biol 88, 669-84.

50. Betzig, E., Patterson G. H., Sougrat R., Lindwasser O. W., Olenych S., Bonifacino J. S., et al. (2006). Imaging intracellular fluorescent proteins at nanometer resolution. Science 313, 1642-5.

51. Kaufmann, R., Schellenberger P., Seiradake E., Dobbie I. M., Jones E. Y., Davis I., et al. (2014). Super-Resolution Microscopy Using Standard Fluorescent Proteins in Intact Cells under CryoConditions. Nano Lett 14, 4171-5.

52. Medalia, O., Beck M., Ecke M., Weber I., Neujahr R., Baumeister W., et al. (2007). Organization of actin networks in intact filopodia. Current biology : CB 17, 79-84.

53. Marko, M., Hsieh C., Schalek R., Frank J. \& Mannella C. (2007). Focused-ion-beam thinning of frozen-hydrated biological specimens for cryo-electron microscopy. Nature Methods 4, 215-7.

54. Rigort, A., Bauerlein F. J., Villa E., Eibauer M., Laugks T., Baumeister W., et al. (2012). Focused ion beam micromachining of eukaryotic cells for cryoelectron tomography. Proceedings of the National Academy of Sciences of the United States of America 109, 4449-54.

55. Villa, E., Schaffer M., Plitzko J. M. \& Baumeister W. (2013). Opening windows into the cell: focused-ion-beam milling for cryo-electron tomography. Current opinion in structural biology 23, 771-7.

56. Rigort, A., Villa E., Bauerlein F. J., Engel B. D. \& Plitzko J. M. (2012). Integrative approaches for cellular cryo-electron tomography: correlative imaging and focused ion beam micromachining. Methods Cell Biol 111, 259-81. 
57. Schaffer, M., Engel B. D., Laugks T., Mahamid J., Plitzko J. M. \& Baumeister W. (2015). Cryofocused ion beam sample preparation for imaging vitreous cells by cryo-electron tomography. BioProtocol.

58. Koster, A. J., Grimm R., Typke D., Hegerl R., Stoschek A., Walz J., et al. (1997). Perspectives of molecular and cellular electron tomography. Journal of structural biology 120, 276-308.

59. Conway, J. F., Trus B. L., Booy F. P., Newcomb W. W., Brown J. C. \& Steven A. C. (1993). The Effects of Radiation-Damage on the Structure of Frozen-Hydrated Hsv-1 Capsids. Journal of structural biology 111, 222-33.

60. Glaeser, R. M. (1971). Limitations to Significant Information in Biological Electron Microscopy as a Result of Radiation Damage. J Ultra Mol Struct R 36, 466-\&.

61. Talmon, Y., Adrian M. \& Dubochet J. (1986). Electron-Beam Radiation-Damage to Organic Inclusions in Vitreous, Cubic, and Hexagonal Ice. J Microsc-Oxford 141, 375-84.

62. Baker, L. A., Smith E. A., Bueler S. A. \& Rubinstein J. L. (2010). The resolution dependence of optimal exposures in liquid nitrogen temperature electron cryomicroscopy of catalase crystals. Journal of structural biology 169, 431-7.

63. Stark, H., Zemlin F. \& Boettcher C. (1996). Electron radiation damage to protein crystals of bacteriorhodopsin at different temperatures. Ultramicroscopy 63, 75-9.

64. Fromm, S. A., Bharat T. A. M., Jakobi A. J., Hagen W. J. H. \& Sachse C. (2015). Seeing tobacco mosaic virus through direct electron detectors. Journal of structural biology 189, 87-97.

65. Bai, X. C., Fernandez I. S., McMullan G. \& Scheres S. H. (2013). Ribosome structures to nearatomic resolution from thirty thousand cryo-EM particles. eLife 2, e00461.

66. Milazzo, A. C., Cheng A. C., Moeller A., Lyumkis D., Jacovetty E., Polukas J., et al. (2011). Initial evaluation of a direct detection device detector for single particle cryo-electron microscopy. Journal of structural biology 176, 404-8.

67. Li, X., Mooney P., Zheng S., Booth C. R., Braunfeld M. B., Gubbens S., et al. (2013). Electron counting and beam-induced motion correction enable near-atomic-resolution single-particle cryoEM. Nat Methods 10, 584-90.

68. Danev, R., Buijsse B., Khoshouei M., Plitzko J. M. \& Baumeister W. (2014). Volta potential phase plate for in-focus phase contrast transmission electron microscopy. Proceedings of the National Academy of Sciences of the United States of America 111, 15635-40.

69. McMullan, G., Clark A. T., Turchetta R. \& Faruqi A. R. (2009). Enhanced imaging in low dose electron microscopy using electron counting. Ultramicroscopy 109, 1411-6.

70. McMullan, G., Faruqi A. R., Clare D. \& Henderson R. (2014). Comparison of optimal performance at $300 \mathrm{keV}$ of three direct electron detectors for use in low dose electron microscopy.

Ultramicroscopy 147, 156-63.

71. Ruskin, R. S., Yu Z. H. \& Grigorieff N. (2013). Quantitative characterization of electron detectors for transmission electron microscopy. Journal of structural biology 184, 385-93.

72. Campbell, M. G., Veesler D., Cheng A. C., Potter C. S. \& Carragher B. (2015). 2.8 angstrom resolution reconstruction of the Thermoplasma acidophilum $20 \mathrm{~S}$ proteasome using cryo-electron microscopy. eLife 4.

73. Voorhees, R. M., Fernandez I. S., Scheres S. H. W. \& Hegde R. S. (2014). Structure of the Mammalian Ribosome-Sec61 Complex to 3.4 angstrom Resolution. Cell 157, 1632-43.

74. Cao, E. H., Liao M. F., Cheng Y. F. \& Julius D. (2013). TRPV1 structures in distinct conformations reveal activation mechanisms. Nature $504,113-+$.

75. Aufderheide, A., Beck F., Stengel F., Hartwig M., Schweitzer A., Pfeifer G., et al. (2015). Structural characterization of the interaction of Ubp 6 with the $26 \mathrm{~S}$ proteasome. Proceedings of the National Academy of Sciences of the United States of America 112, 8626-31.

76. Plaschka, C., Lariviere L., Wenzeck L., Seizl M., Hemann M., Tegunov D., et al. (2015). Architecture of the RNA polymerase II-Mediator core initiation complex. Nature 518, 376-80. 
77. Bartesaghi, A., Merk A., Banerjee S., Matthies D., Wu X., Milne J. L., et al. (2015). 2.2 A resolution cryo-EM structure of beta-galactosidase in complex with a cell-permeant inhibitor. Science 348 , 1147-51.

78. Kuhlbrandt, W. (2014). Biochemistry. The resolution revolution. Science 343, 1443-4.

79. Cheng, Y. (2015). Single-Particle Cryo-EM at Crystallographic Resolution. Cell 161, 450-7.

80. Dubrovsky, A., Sorrentino S., Harapin J., Sapra K. T. \& Medalia O. (2015). Developments in cryoelectron tomography for in situ structural analysis. Arch Biochem Biophys.

81. Gold, V. A., leva R., Walter A., Pfanner N., van der Laan M. \& Kuhlbrandt W. (2014). Visualizing

active membrane protein complexes by electron cryotomography. Nature communications 5, 4129.

82. Hu, B., Morado D. R., Margolin W., Rohde J. R., Arizmendi O., Picking W. L., et al. (2015).

Visualization of the type III secretion sorting platform of Shigella flexneri. Proceedings of the National Academy of Sciences of the United States of America 112, 1047-52.

83. Woodward, C. L., Mendonca L. M. \& Jensen G. J. (2015). Direct visualization of vaults within intact cells by electron cryo-tomography. Cell Mol Life Sci.

84. Xiong, Q. R., Morphew M. K., Schwartz C. L., Hoenger A. H. \& Mastronarde D. N. (2009). CTF determination and correction for low dose tomographic tilt series. Journal of structural biology 168 , 378-87.

85. Yang, Z. F. \& Penczek P. A. (2008). Cryo-EM image alignment based on nonuniform fast Fourier transform. Ultramicroscopy 108, 959-69.

86. Frangakis, A. S. \& Hegerl R. (2001). Noise reduction in electron tomographic reconstructions using nonlinear anisotropic diffusion. Journal of structural biology 135, 239-50.

87. Stoschek, A., Yu T. P. Y. \& Hegerl R. (1997). Denoising of electron tomographic reconstructions from biological specimens using multidimensional multiscale transforms. Int Conf Acoust Spee 27936.

88. Buades, A., Coll B. \& Morel J. M. (2005). A review of image denoising algorithms, with a new one. Multiscale Model Sim 4, 490-530.

89. Fernandez, J. J. \& Li S. (2003). An improved algorithm for anisotropic nonlinear diffusion for denoising cryo-tomograms. Journal of structural biology 144, 152-61.

90. Gilbert, P. (1972). Iterative Methods for 3-Dimensional Reconstruction of an Object from Projections. J Theor Biol 36, 105-17.

91. Batenburg, K. J., Bals S., Sijbers J., Kubel C., Midgley P. A., Hernandez J. C., et al. (2009). 3D imaging of nanomaterials by discrete tomography. Ultramicroscopy 109, 730-40.

92. Crowther, R. A., Derosier D. J. \& Klug A. (1970). Reconstruction of 3 Dimensional Structure from Projections and Its Application to Electron Microscopy. Proc R Soc Lon Ser-A 317, 319-40.

93. Rigort, A., Gunther D., Hegerl R., Baum D., Weber B., Prohaska S., et al. (2012). Automated segmentation of electron tomograms for a quantitative description of actin filament networks. Journal of structural biology 177, 135-44.

94. Martinez-Sanchez, A., Garcia I. \& Fernandez J. J. (2013). A ridge-based framework for segmentation of 3D electron microscopy datasets. Journal of structural biology 181, 61-70.

95. Fernandez-Busnadiego, R., Asano S., Oprisoreanu A. M., Sakata E., Doengi M., Kochovski Z., et al. (2013). Cryo-electron tomography reveals a critical role of RIM1alpha in synaptic vesicle tethering. The Journal of cell biology 201, 725-40.

96. Zhu, Y. X., Carragher B., Glaeser R. M., Fellmann D., Bajaj C., Bern M., et al. (2004). Automatic particle selection: results of a comparative study. Journal of structural biology 145, 3-14.

97. Ortiz, J. O., Forster F., Kurner J., Linaroudis A. A. \& Baumeister W. (2006). Mapping $70 \mathrm{~S}$ ribosomes in intact cells by cryoelectron tomography and pattern recognition. Journal of structural biology 156, 334-41.

98. Chen, Y. X., Hrabe T., Pfeffer S., Pauly O., Mateus D., Navab N., et al. (2012). Detection and Identification of Macromolecular Complexes in Cryo-Electron Tomograms Using Support Vector Machines. 2012 9th leee International Symposium on Biomedical Imaging (Isbi) 1373-6.

99. Fernandez, J. J. (2012). Computational methods for electron tomography. Micron 43, 1010-30. 
100. Martinez-Sanchez, A., Garcia I., Asano S., Lucic V. \& Fernandez J. J. (2014). Robust membrane detection based on tensor voting for electron tomography. Journal of structural biology 186, 49-61. 101. Volkmann, N. (2012). Putting structure into context: fitting of atomic models into electron microscopic and electron tomographic reconstructions. Curr Opin Cell Biol 24, 141-7.

102. Forster, F. \& Hegerl R. (2007). Structure determination in situ by averaging of tomograms. Method Cell Biol 79, 741-67.

103. Frangakis, A. S., Bohm J., Forster F., Nickell S., Nicastro D., Typke D., et al. (2002). Identification of macromolecular complexes in cryoelectron tomograms of phantom cells. Proceedings of the National Academy of Sciences of the United States of America 99, 14153-8.

104. Nickell, S., Mihalache O., Beck F., Hegerl R., Korinek A. \& Baumeister W. (2007). Structural analysis of the $26 \mathrm{~S}$ proteasome by cryoelectron tomography. Biochemical and biophysical research communications $353,115-20$.

105. Hrabe, T., Chen Y. X., Pfeffer S., Cuellar L. K., Mangold A. V. \& Forster F. (2012). PyTom: A python-based toolbox for localization of macromolecules in cryo-electron tomograms and subtomogram analysis. Journal of structural biology 178, 177-88.

106. Chen, Y. X., Pfeffer S., Hrabe T., Schuller J. M. \& Forster F. (2013). Fast and accurate referencefree alignment of subtomograms. Journal of structural biology 182, 235-45.

107. Walz, J., Typke D., Nitsch M., Koster A. J., Hegerl R. \& Baumeister W. (1997). Electron tomography of single ice-embedded macromolecules: Three-dimensional alignment and classification. Journal of structural biology 120, 387-95.

108. Pfeffer, S., Woellhaf M. W., Herrmann J. M. \& Forster F. (2015). Organization of the mitochondrial translation machinery studied in situ by cryoelectron tomography. Nature communications 6, 6019.

109. Stolken, M., Beck F., Haller T., Hegerl R., Gutsche I., Carazo J. M., et al. (2011). Maximum likelihood based classification of electron tomographic data. Journal of structural biology 173, 77-85. 110. Rossmann, M. G. (2000). Fitting atomic models into electron-microscopy maps. Acta Crystallogr D 56, 1341-9.

111. Trabuco, L. G., Villa E., Mitra K., Frank J. \& Schulten K. (2008). Flexible fitting of atomic structures into electron microscopy maps using molecular dynamics. Structure 16, 673-83. 112. Villa, E. \& Lasker K. (2014). Finding the right fit: chiseling structures out of cryo-electron microscopy maps. Curr Opin Struct Biol 25, 118-25.

113. Unverdorben, P., Beck F., Sledz P., Schweitzer A., Pfeifer G., Plitzko J. M., et al. (2014). Deep classification of a large cryo-EM dataset defines the conformational landscape of the $26 \mathrm{~S}$ proteasome. Proceedings of the National Academy of Sciences of the United States of America 111, 5544-9.

114. Kastner, B., Fischer N., Golas M. M., Sander B., Dube P., Boehringer D., et al. (2008). GraFix: sample preparation for single-particle electron cryomicroscopy. Nature Methods 5, 53-5.

115. Cheng, Y., Grigorieff N., Penczek P. A. \& Walz T. (2015). A Primer to Single-Particle Cryo-Electron Microscopy. Cell 161.

116. Cohen, J. (2013). STRUCTURAL BIOLOGY Is High-Tech View of HIV Too Good to Be True? Science 341, 443-4.

117. Shatsky, M., Hall R. J., Brenner S. E. \& Glaeser R. M. (2009). A method for the alignment of heterogeneous macromolecules from electron microscopy. Journal of Structural Biology 166, 67-78. 118. Henderson, R. (2013). Avoiding the pitfalls of single particle cryo-electron microscopy: Einstein from noise. Proceedings of the National Academy of Sciences of the United States of America 110, 18037-41.

119. Nickell, S., Kofler C., Leis A. P. \& Baumeister W. (2006). A visual approach to proteomics. Nat Rev Mol Cell Biol 7, 225-30.

120. Voges, D., Zwickl P. \& Baumeister W. (1999). The 265 proteasome: a molecular machine designed for controlled proteolysis. Annual review of biochemistry 68, 1015-68. 
121. Baumeister, W., Walz J., Zuhl F. \& Seemuller E. (1998). The proteasome: paradigm of a selfcompartmentalizing protease. Cell 92, 367-80.

122. Finley, D. (2009). Recognition and Processing of Ubiquitin-Protein Conjugates by the Proteasome. Annual review of biochemistry 78, 477-513.

123. Beck, F., Unverdorben P., Bohn S., Schweitzer A., Pfeifer G., Sakata E., et al. (2012). Near-atomic resolution structural model of the yeast $26 \mathrm{~S}$ proteasome. Proceedings of the National Academy of Sciences of the United States of America 109, 14870-5.

124. Tai, H. C., Besche H., Goldberg A. L. \& Schuman E. M. (2010). Characterization of the Brain 265 Proteasome and its Interacting Proteins. Front Mol Neurosci 3.

125. Matyskiela, M. E., Lander G. C. \& Martin A. (2013). Conformational switching of the $26 \mathrm{~S}$ proteasome enables substrate degradation. Nature structural \& molecular biology 20, 781-8. 126. Pack, C. G., Yukii H., Toh-e A., Kudo T., Tsuchiya H., Kaiho A., et al. (2014). Quantitative live-cell imaging reveals spatio-temporal dynamics and cytoplasmic assembly of the $26 \mathrm{~S}$ proteasome. Nature communications 5, 3396.

127. Hamilton, A. M. \& Zito K. (2013). Breaking It Down: The Ubiquitin Proteasome System in Neuronal Morphogenesis. Neural Plast.

128. Jansen, A. H. P., Reits E. A. J. \& Hol E. M. (2014). The ubiquitin proteasome system in glia and its role in neurodegenerative diseases. Frontiers in Molecular Neuroscience 7.

129. Sasai, K., Ikeda Y., Tsuda T., Ihara H., Korekane H., Shiota K., et al. (2001). The critical role of the stem region as a functional domain responsible for the oligomerization and Golgi localization of $\mathrm{N}$ acetylglucosaminyltransferase $\mathrm{V}$ - The involvement of a domain homophilic interaction. J Biol Chem 276, 759-65. 


\section{Figure Legends}

Figure 1: Sample preparation and data acquisition workflow for in situ cryo-ET. Arrows point to the subsequent step in the workflow. (a) The cell sample can be provided either in solution (left box), or as an adherent culture grown on top of a gold EM grid (right box). (b) Vitrification methods. Left box: plunge freezing, where an EM grid with cells is attached to a tweezer (left), excess liquid is removed with filter paper (center), and the grid is rapidly plunged into coolant, which can be liquid ethane or a mixture of liquid ethane and liquid propane (right). Right box: high-pressure is used to freeze thicker specimens, including tissue. The cells are immersed in cryoprotectant solutions, such as highmolecular weight dextran and hexadecene, then subjected to $>2000$ bar pressure while being rapidly cooled with a stream of liquid nitrogen. The pressure is then released and amorphous ice is formed within $20 \mathrm{~ms}^{37}$. (c) Top box: correlative microscopy can be used to identify target structures by their fluorescence signal (center, red dots), which aids in locating regions of interest for cryo-FIB milling and cryo-ET (bottom). Bottom box: during cryo-FIB milling, a vitrified cell (top) is eroded with a Gallium ion beam (center, red traces), thinning the sample and making the cell body accessible for cryo-ET observation (bottom). (d) The principle of electron tomography. The vitrified sample (cyan cell) is tilted around a fixed axis (orange line). An electron beam (blue) penetrates the sample and projections images at are recorded through a series of tilt angles (bottom). A tomogram displaying the 3D cellular volume can be reconstructed by aligning and back-projecting these tilt-series images (right).

Figure 2: Image processing workflow for in situ cryo-ET. Each element is connected by either a hashed or solid arrow, indicating an optional or mandatory step, respectively. (a) Denoising can be applied either pre- or post-reconstruction to provide signal enhancement . (b) Segmentation facilitates template matching and analysis of select cellular compartments (colored areas in inset). (c) Template matching and subsequent manual sorting generates a list of possible candidates for the desired macromolecule (illustrated with " $x$ "). (d) Subtomogram averaging initially uses all candidates regardless of conformation or assembly state (differently colored and shaped " $x$ ") to generate an inhomogeneous lower resolution average. (e) Subtomogram classification is capable of separating the dataset into homogeneous subsets, as indicated by the three sorted " $x$ " classes with wellresolved averages. (f) Downstream analysis steps include fitting atomic structures to the subtomogram average and using the positions and orientations from template matching to quantify in situ concentrations, distributions, and interactions. 
Figure 3: Workflow for the in situ characterization of 265 proteasomes in intact neurons ${ }^{9}$. (a) Subtomogram averaging produced a viable average from a randomized initial template within several iterations (from left to right). (b) The previously calculated average was subjected to classification and refinement. From right to left: the variance map (variance in magenta) guided the positioning of two masks used for the classification (green and red circles). This enabled the dataset to be split into assembly state and conformation classes (green and red boxes, respectively). (c) Atomic structure models from single-particle cryo-EM were fit into the two conformation classes with rigid body fitting and cross-validation. Both classes could be assigned to a functional state: the substrate-free ground state and the substrate-processing state.

Figure 4: In situ characterization of Golgi intracisternal protein arrays ${ }^{16}$. (a) Overview slice from a tomogram of a Chlamydomonas Golgi complex, revealing highly ordered membrane-associated complexes between the cisternal membranes. The yellow box is shown enlarged in the inset. Scale bars: $200 \mathrm{~nm}$, Inset: $50 \mathrm{~nm}$. (b) Iterative de novo template generation. The first round of template matching was performed with a single low-pass filtered subvolume as an initial template (top). The resulting average (bottom) was used to initiate the second round of template matching, refining the average. Scale bar: $20 \mathrm{~nm}$. (c) Translational symmetry of the final average (top) was measured by performing an autocorrelation of the volume (center). This translational repeat was then used to generate a final symmetrized average (bottom). The annotated 3D structure shows zipper-like interactions with clear asymmetry across the cisterna from cis to trans (cis-side and trans-side bilayers: light blue and yellow, respectively; luminal protein complexes: magenta, orange, blue, and green). Scalebar: $20 \mathrm{~nm}$. (d) Heatmap displaying the protein array localization from template matching. The arrays clustered in "hot spots", which were only found within the narrow central cisternae regions. Scale bar: $200 \mathrm{~nm}$. (e) Histogram showing protein array orientations from template matching. Most arrays from two separate Golgi reconstructions ("tomogram 1" and "tomogram 2") had the same preferred orientation, with larger luminal proteins projecting from the cis-side of the cisterna (as displayed in the 3D model in panel c). Panels a, $d$, and e are reproduced or modified from ${ }^{16}$. 


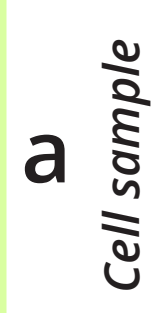

Grown in solution

Grown on EM grid

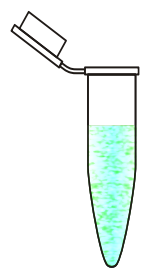

OR
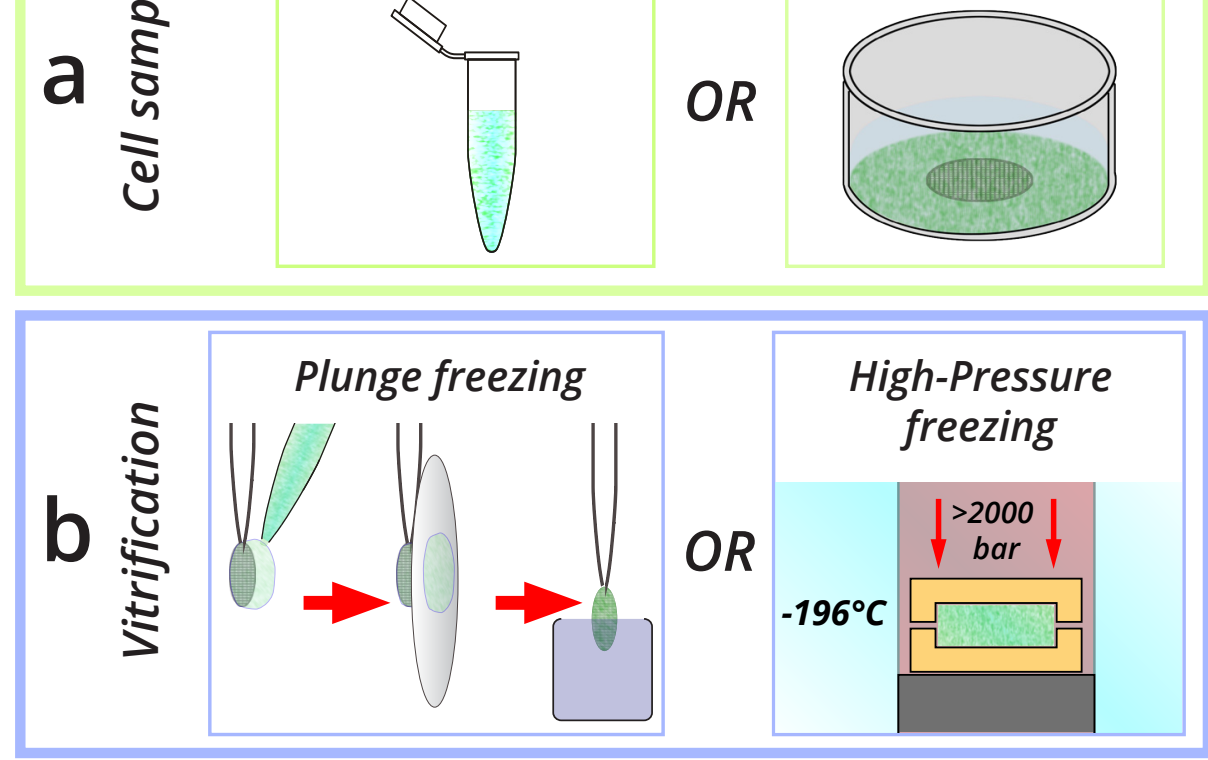

Electron beam

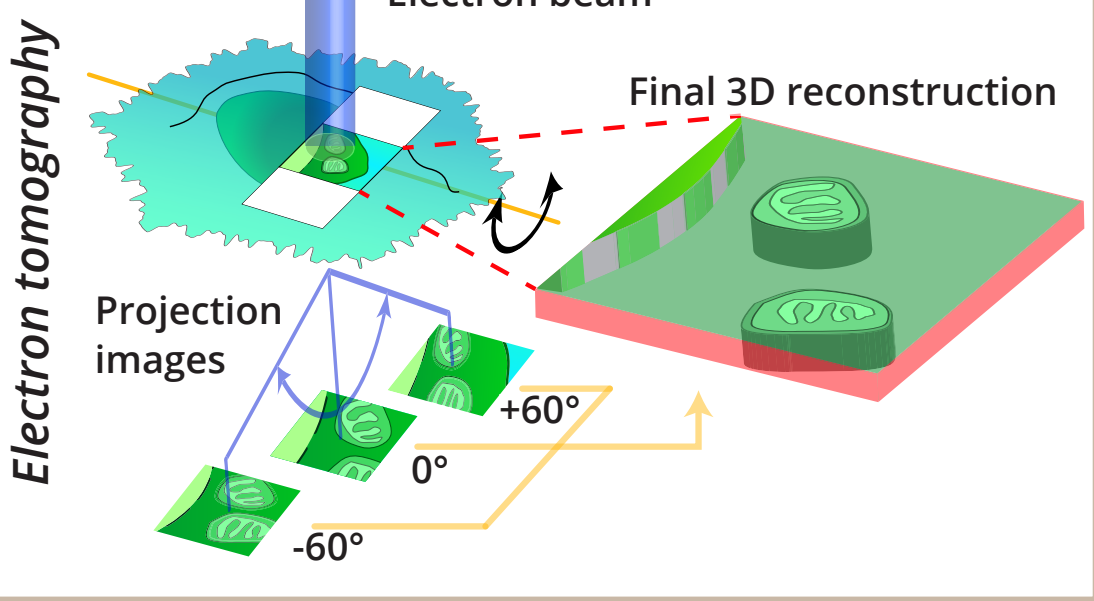

C Optional techniques Correlative microscopy

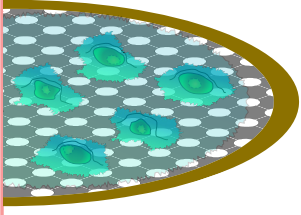

Frozen cells attached to EM grid

Fluorescence microscopy

Correlation with EM data

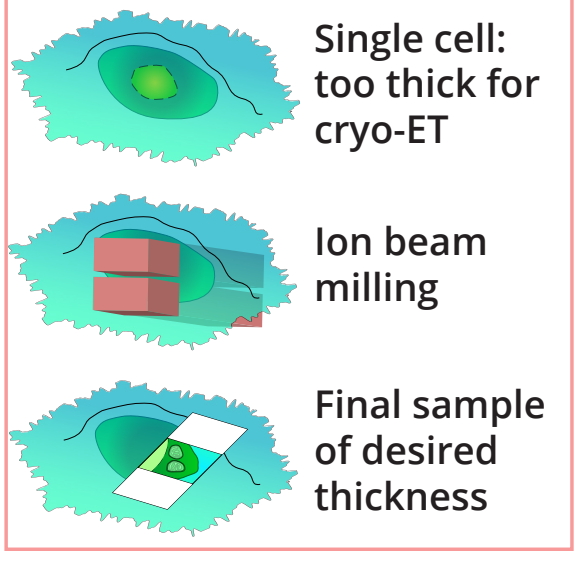

Single cell: too thick for cryo-ET

Ion beam milling Final sample of desired thickness 


\section{Figure 2}

a) Denoising

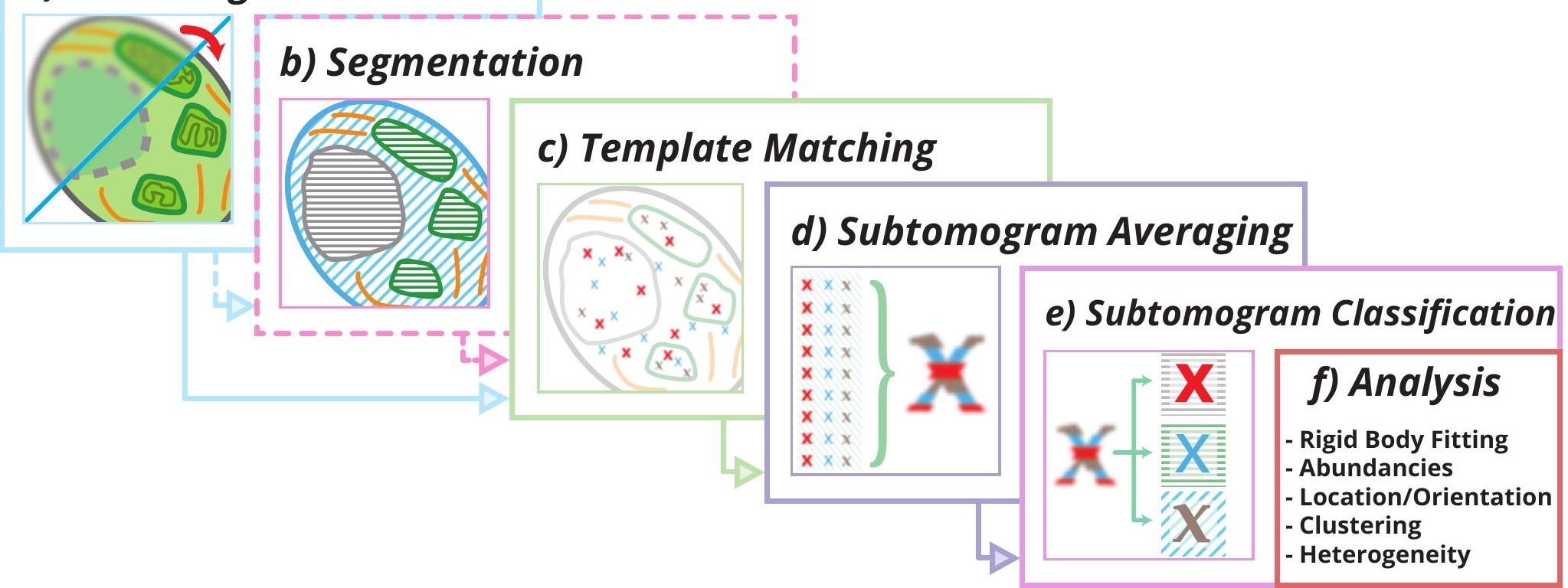


Figure 3

a Subtomogram Averaging

Initial 1st Iter. 2nd Iter. 6th Iter. 8th Iter. 14th Iter. 20th Iter. Reference

\section{b Classification \& Refinement}

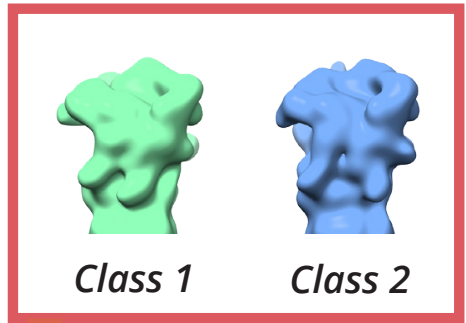

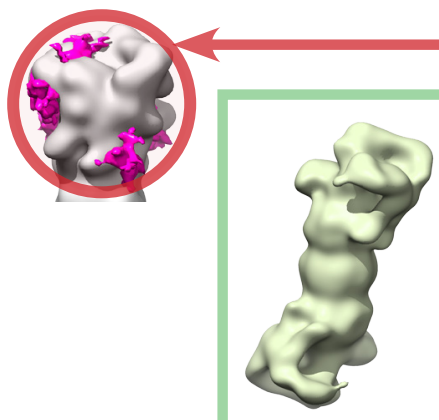

Double

Capped 26S Capped 26S

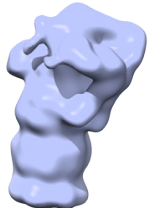

Single

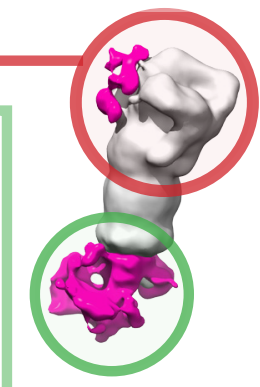

C

\section{Rigid Body Fitting}

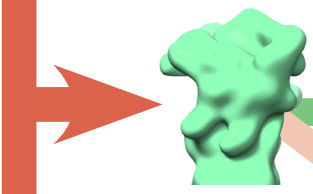

Class 1

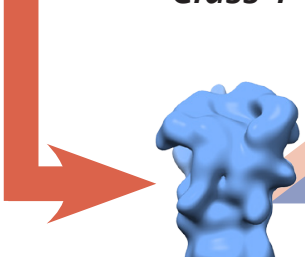

Class 2

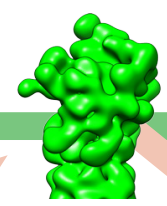

Single Particle Data

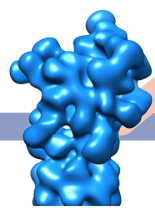

Single Particle Data
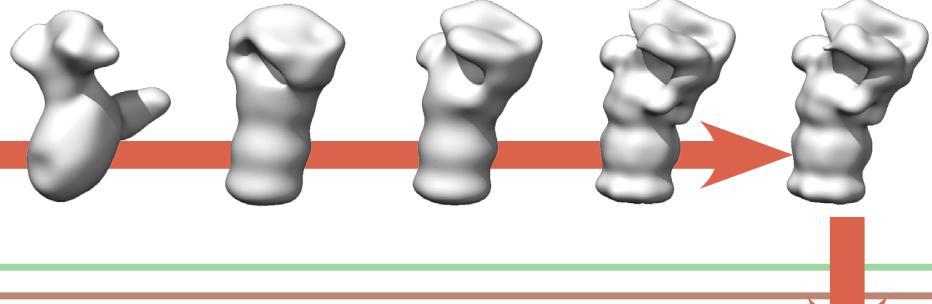


\section{Figure 4}

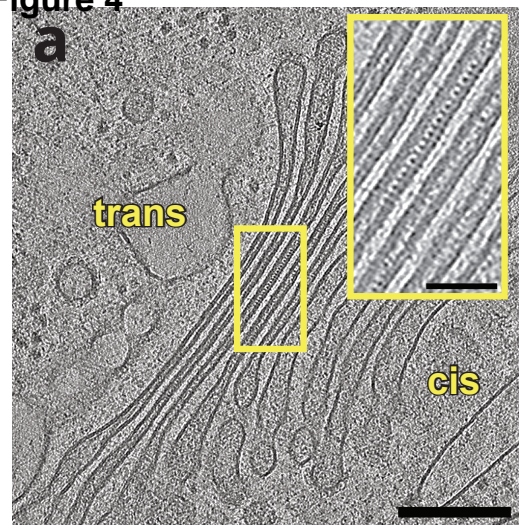

b Initial fitas

Template Matching

Average

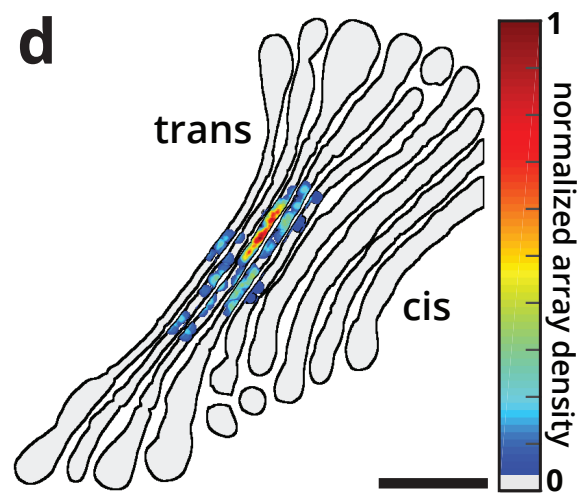

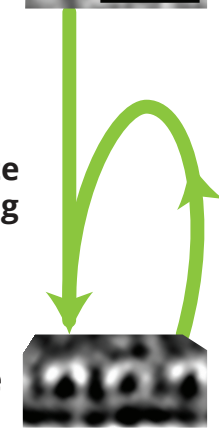

C $\begin{gathered}\text { Final } \\ \text { Average }\end{gathered}$

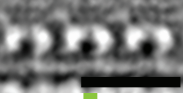

Autocorrelation

Symmetrized Final Average
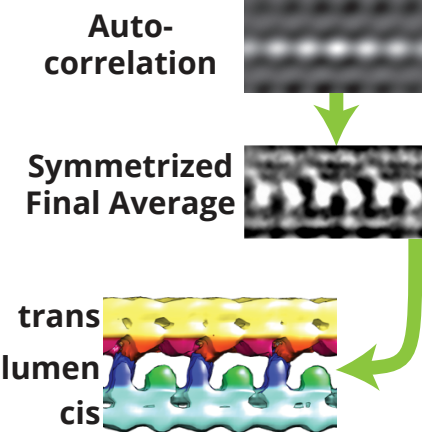

Tomogram1

Tomogram2

e

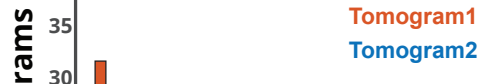

cis

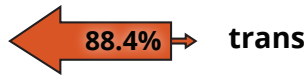

cis

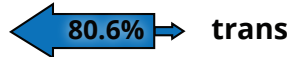

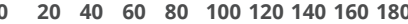
Orientations of subvolumes vs. cis reference $\left[{ }^{\circ}\right]$ 